\title{
What Is Being Used and Who Is Using It: Barriers to the Adoption of Smartphone Patient Experience Surveys
}

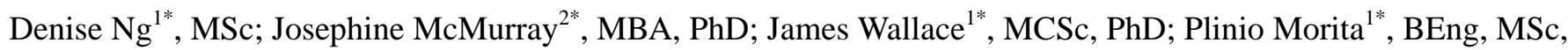
$\mathrm{PhD}$

\footnotetext{
${ }^{1}$ School of Public Health \& Health Systems, Faculty of Applied Health Sciences, University of Waterloo, Waterloo, ON, Canada

${ }^{2}$ Business Technology Management, Lazaridis School of Business \& Economics, Wilfrid Laurier University, Brantford, ON, Canada

*all authors contributed equally
}

\section{Corresponding Author:}

Josephine McMurray, MBA, PhD

Business Technology Management

Lazaridis School of Business \& Economics

Wilfrid Laurier University

73 George Street

Brantford, ON, N3T3Y3

Canada

Phone: 15192427477

Fax: 15197517589

Email: jmcmurray@wlu.ca

\section{Abstract}

Background: Smartphones are positioned to transform the way health care services gather patient experience data through advanced mobile survey apps which we refer to as smart surveys. In comparison with traditional methods of survey data capture, smartphone sensing survey apps have the capacity to elicit multidimensional, in situ user experience data in real time with unprecedented detail, responsiveness, and accuracy.

Objective: This study aimed to explore the context and circumstances under which patients are willing to use their smartphones to share data on their service experiences.

Methods: We conducted in-person, semistructured interviews $(\mathrm{N}=24)$ with smartphone owners to capture their experiences, perceptions, and attitudes toward smart surveys.

Results: Analysis examining perceived risk revealed a few barriers to use; however, major potential barriers to adoption were the identity of recipients, reliability of the communication channel, and potential for loss of agency. The results demonstrate that the classical dimensions of perceived risk raised minimal concerns for the use of smartphones to collect patient service experience feedback. However, trust in the doctor-patient relationship, the reliability of the communication channel, the altruistic motivation to contribute to health service quality for others, and the risk of losing information agency were identified as determinants in the patients' adoption of smart surveys.

Conclusions: On the basis of these findings, we provide recommendations for the design of smart surveys in practice and suggest a need for privacy design tools for voluntary, health-related technologies.

(JMIR Form Res 2019;3(1):e9922) doi: 10.2196/formative.9922

\section{KEYWORDS}

quality of healthcare; surveys and questionnaires; patient satisfaction; data collection; smartphone; mobile phone; risk; privacy

\section{Introduction}

\section{Background}

High-quality patient-centered care is widely recognized as a priority in health care [1] and has been shown to improve patient experience, patient safety, and accessibility to services [2,3]. Health care professionals engaged in patient-centered care focus on ensuring that patients' experiences at a physician, hospital, or rehabilitation facility meet basic standards of care, such as treating them with courtesy, informing them about their care, and minimizing pain during their visit. Standardized questions 
surrounding these aspects of a medical visit are widely used within the health care industry to inform patient-centered care best practices and to improve governance, public accountability, and patient autonomy $[4,5]$.

Paper-based collection of experience surveys remains time-consuming, expensive, and limited by factors such as nonresponse, recall bias, and inadequate sample size [6-8]. Although paper-based surveys may be advantageous in some circumstances, that is, to reduce startup costs or for nondigital natives such as the elderly, the use of smartphone-based survey apps, which we call smart surveys, provides new opportunities to improve data collection techniques. Mobile technology overcomes many of the limitations of paper-based surveys and enables collection of large quantities of real-time data over a broad geographical area. Exploiting this technology opens the possibility of private and public sector services, health care providers, and government bodies effectively engaging with the public, one-on-one, to better respond to their needs. However, at present, there is little guidance to help service providers understand when and where individuals are willing to disclose service experience data using their smartphones. In particular, there is a lack of understanding of users' beliefs, perceptions, and attitudes toward sharing of health service experience feedback using their smartphones.

\section{Patient Experience Surveys}

Patient experience surveys are validated questionnaires, which are developed by health services experts to understand patient perceptions of their health care experience and serve an integral role in patient engagement and service improvement [3]. For example, the WatLX patient experience questionnaire [9-11] determines agreement with statements such as "I was always treated with courtesy," "My physical pain was controlled as well as possible," or "From now on I know what to expect about my care." When such patient experience feedback is collected, collated, and interpreted, findings can drive critical and necessary improvements in service quality, patient safety, and clinical effectiveness $[3,4]$. In recent years, to increase the benefits yielded from such surveys, health service research has begun to shift from "traditional" paper-based methods of survey administration to the use of technology-enabled survey tools.
Yet there is little published research on the use of smartphone technology to collect individuals' experiences of health services.

The health care literature has found no significant differences in data equivalence or validity between paper- and Web-based surveys [12-14]. With mobile devices, researchers are able to collect large quantities of data over broad geographical areas. Furthermore, the use of smartphone-based apps provides access to functionality such as location-based activity detection $[15,16]$ and notifications [17] that can help improve survey compliance and completeness of survey responses to improve the overall reliability of results [18]. In situ assessment of services, where data are collected during or immediately after a service encounter, may yield an even more immediate and insightful level of understanding of service quality [19]. However, the context in which these data are collected can also introduce new issues such as concerns around privacy [20].

Smart surveys, which we define as smartphone survey apps that use advanced functionality to provide more intuitive surveys or gather more contextual complementary data in addition to participant responses, introduce a number of unfamiliar behaviors for patients to undertake that may work as barriers to adoption. For example, they require users to download an app to their personal smartphone and to disclose potentially sensitive information via a digital channel that may be perceived as public or insecure. To better identify and understand how these barriers may impact the adoption of smart surveys, we turn to the theory of perceived risk [21,22].

\section{Perceived Risk Theory}

A user's perceptions of risk can have a negative effect on information system (IS) adoption [21,23,24]. Initially introduced in the context of consumer behavior research, perceived risk can be conceptualized as the subjective expectation of loss experienced by a consumer during purchase decisions [21]. Perceived risk describes risk as a multidimensional construct that comprises facets such as financial risk, performance risk, physical risk, psychological risk, social risk, time risk, and privacy risk. Our framework of perceived risk is adapted from studies by Jacoby and Kaplan [22] and Featherman and Pavlou [23] (Table 1).

Table 1. Seven dimensions of perceived risk framework.

\begin{tabular}{|c|c|}
\hline Risk dimension & Definition \\
\hline Performance & $\begin{array}{l}\text { The possibility that a product or service is not performing the way it was designed or advertised, therefore failing to deliver } \\
\text { the expected benefits. }\end{array}$ \\
\hline Financial & $\begin{array}{l}\text { The possibility that the use of a product or service will cause undesired financial loss (due to purchase and incurring fees or } \\
\text { fraud). }\end{array}$ \\
\hline Time & $\begin{array}{l}\text { The possibility that a product or service will cause the consumer to lose time from researching the product, learning the use, } \\
\text { or returning the product if it underperforms. }\end{array}$ \\
\hline Psychological & $\begin{array}{l}\text { The risk that the purchase or performance of a product or service will cause a negative effect on the consumer's mind or self- } \\
\text { perception (eg, frustration or loss of self-esteem). }\end{array}$ \\
\hline Social & The potential loss of the consumer's social circle due to the use of a product or service. \\
\hline Physical & The possibility that the use of a product or service may be harmful or injurious to the consumer's health. \\
\hline Privacy & The potential for personal information being shared without consent and/or used for purposes other than originally intended. \\
\hline Overall & A general measure of perceived risk when all criteria are considered together. \\
\hline
\end{tabular}


Consumer behavior and IS research has found perceived risk and its antecedents to be key predictors of electronic service adoption; for example, perceived risk and its dimensions are inhibitors of technology acceptance model variables [19]. Of all the facets of perceived risk, privacy (security) risk is demonstrated to be the most important barrier in the adoption of e-services for consumers, having both direct and indirect influences on the intention to adopt [25]. Financial risk, the second most important inhibitor to adoption, also has a significant negative impact on attitude to technology adoption [26]. Time risk has a negative influence on attitudes, implying consumers are concerned about delays and length of time to complete a transaction.

Perceived risk impacts attitudes toward adopting mobile e-services [27-29] as well as the intentions of use among both frequent and infrequent users of mobile e-services. In these studies, results have consistently shown overall perceived risk to be mediated by privacy, financial, time, and performance risks. With respect to mobile health (mHealth) app adoption studies, perceived risk has significant and negative effects on attitudes toward adoption. For example, Schnall et al [30] identified patient concerns regarding security (eg, health information or location sharing) when referring to mHealth apps and smartphone devices, similar to Zhou [31].

Previous research has categorized users based on the intensity of their perceptions of privacy risk. Westin [32] separates technology users into 1 of the 3 risk groups based on their willingness to share personal information on the Web: (1) privacy fundamentalists (high privacy orientation and supports regulatory controls), (2) privacy pragmatists (weigh benefits to self or society and bases trust on context), and (3) privacy unconcerned (willing to share information and reject privacy concerns). These categories were developed over a series of more than 30 consumer surveys [33] and have been used extensively by the computer-human interaction community as a requirements engineering design tool to help anticipate user needs and design functionally relevant technology. However, in practice, this methodology has faced criticism, and research has found a lack of correlation between Westin's categories and user behaviors and attitudes (ie, willingness to share information), perhaps attributable to the development of the questions before the internet [34-36]. In response to this mismatch between Westin's categories and user behavior and to more fully account for the importance of privacy risk as a barrier to adoption of smart surveys, we turn to Dupree et al's work on privacy personas $[37,38]$

\section{Privacy Personas}

Dupree et al [37] developed privacy personas to add contextual information to participants clustered by their attitudes and behaviors related to security and privacy. The personas provide a better understanding of a user's proactivity and ability to act upon privacy risk concerns, better aligning their behavior with their attitudes. Dupree et al identify the following 5 clusters:

1. Fundamentalists (high knowledge and motivation): like Westin's privacy fundamentalists [33], these individuals are highly concerned with privacy and show distrust toward corporate monitoring. They exercise extreme caution when handling their information, often encrypting their devices.

2. Lazy experts (high knowledge and low motivation): these individuals share the same technical knowledge as fundamentalists, but often choose convenience over security and socialization over privacy. They continue to put effort into protecting their privacy, however not to the extent where they would limit their interactions with society.

3. Technicians (medium knowledge and high motivation): have less technical knowledge compared with the fundamentalists and lazy experts. However, they show limited trust in privacy settings and are highly motivated to protect their privacy, often choosing privacy over being social. They tend to form their attitudes more intuitively but will change their behavior when provided with evidence.

4. Amateurs (medium knowledge and medium motivation): these individuals are just learning about security concepts. They are not nearly as motivated or knowledgeable as the other previously mentioned groups. Despite having limited knowledge, this group will still act to protect themselves from privacy threats.

5. Marginally concerned (low knowledge and low motivation): with limited knowledge about security concepts, they trust networks and websites which claim to be safe. They are aware of potential privacy threats but feel these threats are unlikely to happen to them.

Morton and Sasse [39], who performed their research concurrently with Dupree et al, also identify 5 clusters that closely correspond to those listed above but in the context of location disclosure.

The purpose of this study was to understand what beliefs, perceptions, and attitudes influenced patients' intentions to share health service experience feedback using their smartphones, in particular, what role perceived risk plays in this process. Health care providers are increasingly being held accountable for the quality of services they provide; however, data collection is expensive, response rates are low, and turnaround times can be long. Although mHealth apps are common in the sector, and smartphones have been used to collect experience data in other industries [40,41], there has been little research into the use of mobile apps to collect in situ, location-based experience data in health care.

\section{Methods}

\section{Participant Recruitment and Selection}

We recruited participants from a local university between January and February 2017 using posters, email, and snowball sampling techniques. Participants were classified according to their privacy persona and their dimensions of perceived risk, and their responses were sequentially analyzed to allow researchers to evaluate the breadth of our sample and to ensure that individuals with different technical backgrounds as well as varying degrees of privacy tolerance related to information sharing were included in the study. Recruitment and analysis proceeded until saturation $[42,43]$. Participants received Can $\$ 10$ for participating in the interview. 
Figure 1. MetricWire's smartphone app served as a platform to administer the patient experience SmartSurvey.

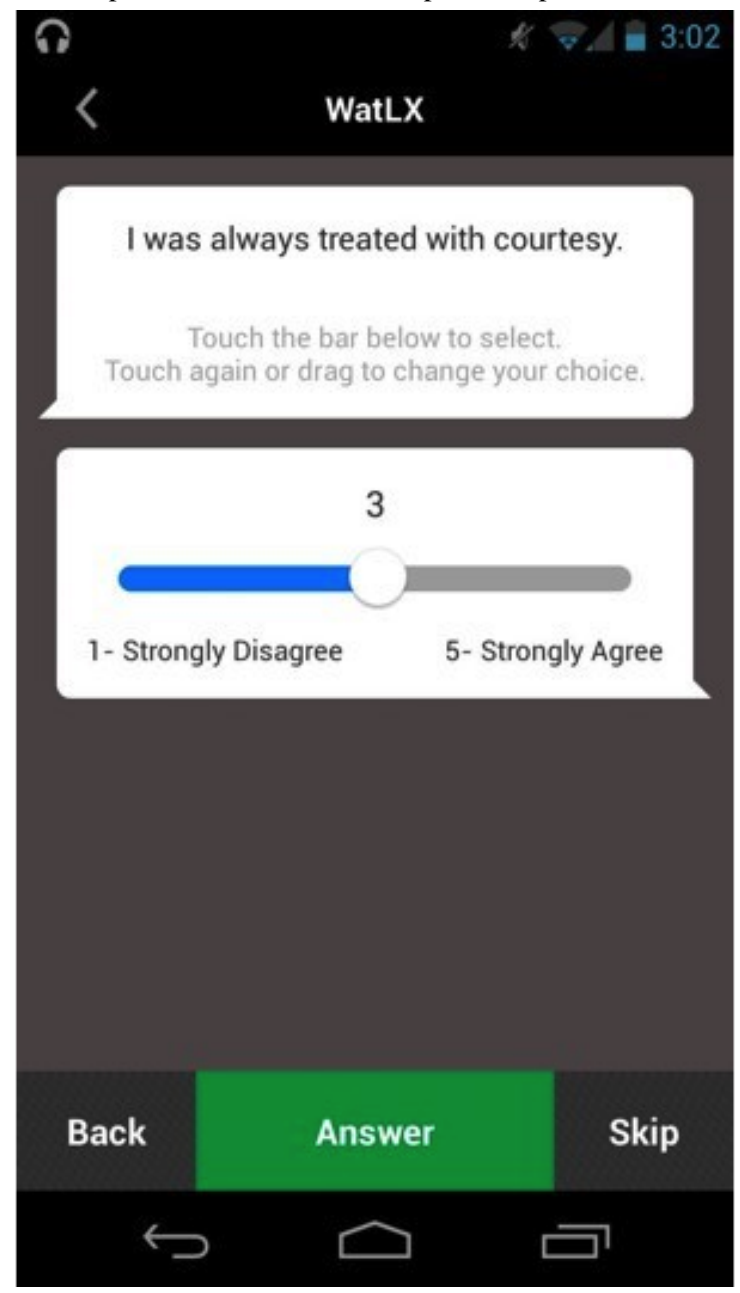

\section{Data Collection and Analysis}

Participants were welcomed upon their arrival and were given an overview of the purpose of the study and the data collection process by a researcher. Participants reviewed and signed an informed consent form and provided demographic information. Data were collected from participants using short questionnaires followed by a semistructured, in-depth interview. The information gathered from the questionnaires and think-aloud technique provided information for classification of participants into privacy persona clusters as well as complementary data for further context.

In the first questionnaire, participants were asked to rate their perspectives on privacy and security (PAS) (Multimedia Appendix 1). The second questionnaire included Westin's privacy user questions [32] and questions that were related to their knowledge and motivation to protect their privacy (Multimedia Appendix 2) [37]. Before completing the final questionnaire and interview, participants were given the opportunity to learn more about the mobile app used for the survey distribution, analysis, and administration [44] and to evaluate its key features and user interface. The MetricWire app is a commercially available mobile phone and Web-based survey platform [44]. The app allows mobile completion of surveys, with functionality such as automatic alerts or triggers to prompt phone owners to respond to a survey based on factors such as time of day or location of the device. Researchers loaded a modified standardized and validated patient experience survey $[9,10]$ into the MetricWire platform using a smartphone provided by the researchers (Figure 1). Participants were asked to recall their last visit to a physician and fill out the survey. Completion of the survey ranged from $2 \mathrm{~min}$ to $5 \mathrm{~min}$.

Using cognitive interviewing techniques [45] (think aloud), where questions are administered, and participants are encouraged to verbalize the reasoning behind their answers, a third questionnaire (including questions adapted from Jacoby and Kaplan's [22] perceived risk study), was used to assess participants' perceptions of risk (Multimedia Appendix 3). These semistructured participant interviews ranged from 20 min to $40 \mathrm{~min}$. Throughout and at the end of the interview, the researcher summarized their interpretation of each participant's responses. Participants were encouraged to add any additional information that they felt was missing from the summarized interview responses. This process served as an informal method for member checking [46].

Upon completion of each participant interview, participant responses were transcribed manually from the digital recordings and thematically analyzed using QSR International's NVivo 11 [47]. Responses were used to inform subsequent interviews. We used constant comparison and content analysis to code and analyze the transcripts [48], with 3 researchers (DN, JM, and JW) reviewing the interview transcripts independently and using 
consensus methods to iteratively discuss content and discrepancies to ensure coding consistency. Data were manually sorted using the perceived risk framework and then thematically analyzed to uncover the unique challenges facing smart surveys' adoption and use for patient experience sampling. Interview transcripts underwent initial open-ended coding where quotes were divided into 4 concepts and 12 subconcepts based on similarities in meaning or context. These concepts were then discussed among the researchers to further develop emergent themes. The themes were developed based on both the perceived risk typology (deductive reasoning) and open and axial coding of interview transcripts (inductive reasoning) [47,49].

To assign participants to 1 of Dupree's clusters, each participant's data were reviewed (DN), and the participant was preliminarily assigned. Following a process of discussion (JM, JW, and PM), participants were reassigned as necessary until each cluster remained stable. Participant data were collected until we had at least one participant from each of the Dupree clusters identified, and saturation was achieved, where no new themes or evidence emerged from the interview transcripts $[42,43]$.

Ethics approval for this study was sought and obtained jointly from the ethics committees at Wilfrid Laurier University and the University of Waterloo (\#4690). All participants provided written informed consent before participating in the study.

\section{Results}

\section{Overview}

We conducted 24 semistructured interviews with Canadian smartphone owners (7 male and 17 female) with varying educational backgrounds, technical knowledge, and motivations to protect privacy. All the participants were registered university students, half at the graduate level and, as such, are "digital natives" and thus confident using smartphones and mobile apps [50]. All had received some postsecondary or postgraduate education, were comfortable speaking English, and regularly used a smartphone. The average age of the sample was 23.9 years (minimum: 21 years, maximum: 56 years, and median: 22.5 years)

\section{Perceived Risks and Privacy Personas of the Sample}

Respondents were classified according to Dupree et al's [37] privacy clusters framework (Table 2). Participant 4 was rated with low technical knowledge yet high motivation to protect their privacy. Therefore, they did not fit any of the Dupree privacy personas, and we classified them as "Undefined."

To develop an understanding of the core issues facing smart survey adoption, we also categorized responses according to the dimensions of perceived risk [21,22] (Table 3). Participants were most concerned about PAS and performance risk; under the circumstances, that is, the introduction of a novel app for use in a contextually sensitive location, this result was predictable. None of the participants expressed concerns related to their psychological or social well-being as a result of using the app.

Table 2. Participants classified by privacy persona.

\begin{tabular}{llll}
\hline Privacy persona & Knowledge & Motivation & Statistics, $\mathrm{n}(\%)$ \\
\hline Marginally concerned & Low & Low & $8(33)$ \\
Technicians & Medium & Medium & $7(29)$ \\
Amateurs & Medium & Low & $5(21)$ \\
Lazy experts & High & Low & $2(8)$ \\
Fundamentalists & High & High & $1(4)$ \\
Undefined & Low & High & $1(4)$ \\
\hline
\end{tabular}

Table 3. Number of participants who classified dimensions of perceived risk as either "likely" or "very likely."

\begin{tabular}{ll}
\hline Type of perceived risk & Statistics, $\mathrm{n}(\%)$ \\
\hline Privacy and security & $18(75)$ \\
Performance & $12(50)$ \\
Time & $4(17)$ \\
Financial & $2(8)$ \\
Physical & $1(4)$ \\
Psychological & $0(0)$ \\
Social & $0(0)$ \\
\hline
\end{tabular}




\section{Thematic Analysis}

A number of themes emerged from our analysis of cognitive and in-depth interview transcripts: (1) perceived risks associated with smart survey use, (2) loss of information agency, and (3) trusted data collectors and altruistic intentions. These are organized according to the focus of this study: first, how perceived risk impacts the propensity to use smartphones to provide service feedback using our perceived risk framework, and second, the role of participants' key beliefs, perceptions, and attitudes in that process.

\section{The Impact of Perceived Risk on Intentions to Use Smart Surveys}

\section{Performance Risk}

Although performance risk was the most cited type of risk, participants perceived it to be minimal when downloading or using smart surveys. The likelihood of performance risk was rated by $25 \%$ (6 out of 24) participants as "Very Unlikely" and as "Unlikely" by 46\% (11 out of 24) participants. Some participants attributed this lack of risk to smart surveys being more simplistic in design than other apps on their phone and others to functionality that allowed participant audit before data were submitted:

\section{...from my point of view, it doesn't look too fancy or a gaming application with a lot of coding and stuff...I feel like chances of it not working... will be low. [P21, Amateur] \\ It's not quite as advanced as some other apps. And since it submits [data] all at once... I would be able to look at all the information before it's submitted. [P14, Lazy expert]}

\section{Time Risk}

Some participants commented that smart surveys' voluntary nature mitigated any associated risks related to time. Others disagreed, saying time loss from downloading and using the app was "very likely"; as they perceived that only health care providers would ultimately benefit from the data, they saw no off-setting personal benefit to mitigate the time risk:

Very likely, because it does benefit just the company, not really yourself. And like I said, it already takes a long time as an app it downloads and all that stuff... [P4, Undefined]

\section{Financial Risk}

The majority of participants felt that the possibility of financial loss associated with the app was either "Very Unlikely" $71 \%$ (17 out of 24 participants) or "Unlikely" $21 \%$ (5 out of 24 participants). The perception of low financial risk was attributed to smart surveys' free download and lack of request for any financial information:

As a patient, would I have to pay money to download the app?...In this case, there doesn't seem like there's any chance that I would be losing money with Smart surveys. I don't think it's asking for credit card information or anything. [P22, Lazy expert]

\section{Psychological Risk}

When asked to judge their perception of psychological risk associated with smart surveys, nearly all participants 8\% (20 out of 24) rated their perceived psychological risk to be "Very Unlikely." Participants were familiar with providing feedback and with using smartphone apps:

...it's voluntary if there was something I didn't want to say or discuss, I wouldn't have taken it. [P21, Amateur]

\section{Social Risk}

Overall, participants perceived a very low possibility of social risk, noting that completing surveys on a smartphone was sociably acceptable and could be completed privately:

I'm on my phone a lot anyways. I'm answering surveys. I don't think anyone would think of me differently because it's just surveys. [P7, Technician]

\section{Physical Risk}

There was little to suggest that participants perceived any physical risk associated with this technology and noted that it was comparable with any other app on their smartphone:

Well, it's just filling out buttons on a survey. I don't think there should be health issues any more than health issues from just using a smartphone. [P7, Technician]

\section{Privacy and Security Risk}

In line with the 6 dimensions of risk, participants rated the likelihood of "overall" risk associated with smart surveys as low. However, when discussing the overall risk, the predominant concerns related to PAS risk included the loss or misuse of sensitive information associated with their location and activity:

Personally, I don't like the idea of data being collected on me...If there's an app that could literally tell you physically where you're being, that's part of the metadata government can collect on you. [P12, Technician]

Similarly, participants displayed heightened sensitivity and apprehension about the possibility of the app being used to retrieve additional information unrelated to the research:

\section{Maybe if I download some app, maybe someone can get your personal information on your phone. [P6, Marginally concerned]}

Moreover, 1 participant was concerned that a third party, such as an employer or insurance company, could use the collected data to deny individuals employment or insurance claims:

If it's not associated with my insurance company in any way, and it's only for the health care to improve their staff's interaction with their patient. I don't think it would be likely [I would consider it a risk]. [P24, Technician]

Yes. I just think I would just want to know what is being used and who's using it. And if someone could tell me that, then it might change my mind from not 
giving out that information to giving information. [P11, Marginally concerned]

I don't consider the information to be very sensitive. Even if it does go into the wrong hands, which would be weird, I probably wouldn't mind too much. [P7, Technician]

Another expressed a belief that mobile apps may be less secure than traditional desktops apps and that the use of smartphones introduces risks such as susceptibility to hackers, in-device vulnerability, and susceptibility to loss:

It's not $100 \%$ safe...I'm not sure apps interact with each other in a smartphone...if other apps can steal information from another app. It's not $100 \%$ safe. [P21, Amateur]

I think it's safe. It's not risky to share feedback. But you never know. Sometimes people can get your secure passwords, your bank passwords. [P23, Amateur]

...it's not really safe to send it through the smartphone... a smartphone can easily go into the wrong hands. It could get stolen, or even borrowed, maybe you just left it somewhere... [P7, Technician]

Location (Global Positioning System, GPS) data were an area of particular sensitivity. The majority of participants $71 \%$ (17 out of 24) were reluctant to disclose their location (GPS) data for service quality improvement. Many chose to not share location information for reasons of privacy, safety, and battery life:

If it's on all the time, I feel like someone's following me all the time or someone can see that they're following me and it probably drains out my battery too. [P13, Amateur]

Other issues included concern over the perceived lack of standards surrounding the handling and storage of patient experience data. The heightened sensitivity was not surprising given the considerable attention to PAS risks associated with mHealth apps [51] and frequent breaches of health ISs in the free press at the time. Finally, the overall usability of smartphones relative to desktops was also considered, reflecting limitations of a smartphone's display as part of their confidence in the device to live up to the task of completing a survey:

...I mean, it's kind of normal for me to do surveys on a computer but doing it on the phone is a little awkward. Another reason, I guess, I'm not too fond of reading too much on a smartphone 'cause I have a smaller screen and the text is small. [P7, Technician]

\section{The Role of Participants' Beliefs, Perceptions, and Attitudes}

\section{Trusted Data Collectors and Altruistic Intentions}

Importantly, the third-party mobile app for data collection using a smartphone was perceived as distinct from the health care facility requesting the data, which participants generally trusted to comply with ethical treatment of their data:

\section{...because it's health care facility. I have complete trust in them. [P2, Amateur]}

The identity of those who receive and interpret patient experience data was an important consideration for participants when deciding to complete a patient experience smart survey. More than half the participants $6 \%$ (14 out of 24) mentioned concerns over who received and viewed their information. Knowing who the users were and how the data would be used helped them decide whether or not to share feedback. Sharing experience data with their care providers was not a barrier, given its less sensitive nature, and thus diminished consequences if mishandled.

For some participants with altruistic intentions, the impact of their feedback was a significant factor in decision making, particularly where trusted care providers might directly or indirectly use that feedback to improve service quality for others:
I want my feedback to improve the service. I don't write my feedback for someone who can't change anything or improve anything. [P20, Marginally concerned]
If I share my data with the doctor, the administrator will not benefit me if they look into my data. Anyone who's not really involved with the service. If I want to share my information in my smartphone, I want to give it to the doctor directly...It's also the benefit of the smartphone, it can give it directly to the doctor. [P15, Marginally concerned]

\section{Loss of Information Agency}

Participants expressed concern that collected data may be used for purposes beyond what was initially intended or disclosed, specifically that it might affect their "information agency." This differed from their privacy concerns, where privacy risk is defined as the potential loss of personal information without the consumers' knowledge following the use of a service or a product [23]. The loss of information agency is the loss of control over the interactions after the information has been shared by the participant. An example of loss of information agency might relate to receiving marketing emails following a service encounter, using contact information a patient provided to an endorsed third party collecting a trusted provider's service quality survey data. In addition, 1 participant explained that the loss of agency can be apparent when seeing Web-based advertisements tailored to her daily life:
Maybe it's stuff you don't necessarily want a third party to know and they do know it because sometimes certain third party companies display ads based on what you've done if you see an ad that's something related to you in story that you've done. [P21, Amateur]

In general, the participants exhibited comments and knowledge consistent with their Dupree classification. 


\section{Discussion}

\section{Principal Findings}

Mobile apps are increasingly being used to gather real-time clinical and ecological patient data and to help manage workload in the health services sectors [50-54]. Although smartphones are changing the way we deliver health care and engage patients [55], examples of their use in collecting in situ patient service experience data are scant. We explored perceptions and attitudes, which impact the adoption and use of smartphone-based apps to collect patient experience data, referred to as smart surveys. The theory of perceived risk [21,22] suggests there will be inhibitors to smart surveys adoption. Yet, although participants mentioned perceived risks normally associated with electronic commerce and other Web-based activities such as social, psychological, physical, financial, and time risks $[23,56]$, these were considered minimal.

The study used an app that participants would have to download, retain on their phone, and manage alerts and the software app itself over time. The most commonly perceived risk was PAS, consistent with other mHealth and wearables literature $[51,57,58]$. Participants' PAS risk perceptions were related to connectivity, data sharing, encryption, and storage. Consistent with Dupree et al [37], we observed that not all digital natives have the same level of technical knowledge. Our thematic analysis revealed other factors indirectly related to risk that influenced participant's perceptions of smart surveys as a conduit for patient service experience data collection. In particular, participants trusted the data collectors and communication channel, thereby reducing perceptions of risk. Conversely, concerns over loss of information agency, evoked based on past social and personal experiences where they lost control of their data, served to amplify their PAS concerns. These themes have implications for the design and use of smart surveys apps in practice in 3 main areas. The 3 themes, with recommendations for developers are as follows:

\section{Support Communication Between Providers and Recipients of Information}

When individuals do trust their health care provider, the presence of trust reflects a belief that the provider has the ability and motivation to make changes that result in service improvements [59,60]. However, existing surveys often do not allow for participants to see the impact of their feedback or for providers to acknowledge its importance. Participants' concerns revealed a need to better communicate patient experience survey goals to patients and to ensure that feedback impacts service improvements. Only half of the participants believed their feedback was important, and some participants regarded feedback as a formality rather than a tool to improve services. Similarly, we found that participants wanted health care providers who receive their comments to have the authority to implement changes. Our results point to an opportunity for smart surveys' functionality to be expanded to include frictionless feedback loops where health care providers acknowledge the importance of participation and communicate when service feedback has been received and implemented. These communications are essential in building trust between patients and providers and are poorly supported through paper surveys.

Furthermore, our results suggest a need to better identify any complementary uses and recipients of survey data. Research ethics standards of practice require that researchers inform participants how data will be used at the beginning of a survey; however, this is not necessarily the case for private or nonprofit health service providers. Outside of personal health information, the use and management of which is often governed by legislation, consumers have very little control over what data are stored and shared for commercial use [61]. Our results suggest that participants want health care providers to affirm who and how the feedback data will be used and its sequelae.

\section{Recommendation Number 1}

Smart surveys functionality should foster trust between patients and providers by identifying the recipients of feedback data and communicating when it is read and what improvements to care are made as a result.

\section{Provide Transparency of Motives and Options}

Participants perceived a lack of confidence in the security of smartphones and that they can be perceived as a second-class computing device when compared with desktop personal computers for completing surveys. For example, participants expressed concerns about installing apps on their personal devices and uncertainty about how data may be shared between different apps. This was notable among the amateurs and marginally concerned. For individuals with more in-depth technical knowledge such as the fundamentalists, disclosure of implementation details was equally important, such as the types of permissions the app required, the type and location of servers on which the data would be stored, whether information would be encrypted, and the length of time their information would be retained. However, it should be noted that this was the exception. As with Gkioulos et al, we found that digital natives tended to ignore or were complacent about privacy policies [50].

\section{Recommendation Number 2}

For technical users, provide optional information about where data will be stored, for how long, and whether it will be encrypted.

\section{Recommendation Number 3}

Where possible, smart surveys should provide optional modalities to complete experience surveys on devices other than smartphones.

\section{Controlling Access to And Sharing of Information}

Although there were disagreements about the sensitivity of feedback data, participants were consistently hesitant about unauthorized use of or access to data, particularly their location (ie, GPS) [16,62]. Participants believed that location data introduced a higher level of risk and loss of agency that could lead to subsequent inconveniences (eg, telemarketing interactions) or consequences (eg, identity theft). In some cases, participants believed that loss of information agency could lead to the loss of privacy, loss of finances, or physical harm. 
The perceived risk of losing agency represents a significant barrier to adoption of "advanced" smart surveys features such as geofencing (use of technology to create a virtual geographic boundary that triggers or alerts when a mobile device enters or leaves the area). For example, smart surveys can reduce recall bias by prompting patients for feedback soon after they leave a physician's office, instead of days or months later in traditional survey methods. The majority of participants found location-based prompts too intrusive and risky and had location services disabled on their phone. This finding is consistent with prior research that demonstrates concerns for privacy are higher when the service is based on tracking the user's location [62]. Furthermore, participants were conscious of the consequences of their location being disclosed to third parties.

\section{Recommendation Number 4}

Smart surveys should support alternatives to location services for prompting patients for feedback, for example, quick response codes or calendar integration.

\section{Limitations}

The themes identified through our interviews helped to develop an understanding of barriers to smartphone-based patient experience surveys. Nevertheless, we are careful to acknowledge limitations to this study. First, the attitudes and perceptions of risk held by our participants were captured at 1 point in time, and attitudes toward adoption can change over time and as the y become more familiar with technology $[63,64]$. Furthermore, participants were not asked to download and use the app on their own devices. Consequently, there may have been less consideration given to risk as participants did not actually surrender any personal information. Future work will address these limitations through data triangulation and a longitudinal validation that patient behaviors reflect their reported perceptions. Second, participants were mostly younger (average age: 23.9 years) and more educated, and as a result, they are not necessarily representative of the largest patient segment using health care services [65]. Although the results of this study may not be generalizable to the wider population, a majority of adults now own cell phones, $77 \%$ of them own smartphones, and a growing percentage of adults aged 50 to 64 years and over 65 years are smartphone users (73\% and $46 \%$, respectively)
[66]. These rapidly growing rates and the decreasing availability of cell phones without expanded digital capability and access to the internet suggest that adults across the life stage will soon experience similar issues as the study sample.

We used nonprobability convenience sampling and a nonsystematic recruitment process for this exploratory study; we did not anticipate our findings would be exhaustive; however, we believe that they add to the understanding of this emerging domain. On the basis of our findings, we believe that individuals with higher technical knowledge and motivation to protect their privacy were under-represented. Finally, the strength of qualitative research is its ability to describe and understand both obvious and latent phenomena contained within the "thick descriptions" provided by interview data. Although our interpretation of these exploratory data is nongeneralizable, the use of in-depth interview methodology provides researchers with an appreciation of the complexity and context of this relatively new research domain. It should also be acknowledged that with every new innovative technology, the patterns of risk and security concerns may differ from those of ostensibly similar legacy systems [24].

\section{Conclusions}

The use of smartphone-based patient experience surveys provides new and exciting opportunities for health care providers to assess and improve the quality of health services. We conducted 24 semistructured interviews with smartphone users to explore the types of perceived risks that may exist when using smart surveys in the context-sensitive health services sector. The results demonstrate that the classical dimensions of perceived risk raised minimal concerns for the use of smartphones to collect patient service experience feedback. However, PAS risk associated with trust in the doctor-patient relationship, the reliability of the communication channel, and the risk of potential loss of agency over shared information may inhibit adoption. Conversely, altruistic motivations to contribute to health service quality for others may facilitate patients' adoption of smart surveys. We conclude that barriers and enablers of adoption of novel technologies may change from sector to sector and should be further explored.

\section{Acknowledgments}

This work is funded by a Social Science and Humanities Research Council of Canada Insight Development Grant (\#430-2016-00858). We would like to thank Mr Brian Stewart and the team at MetricWire for their support of this study.

\section{Conflicts of Interest}

None declared.

\section{Multimedia Appendix 1}

Questionnaire: participant perspectives on privacy and security.

[PDF File (Adobe PDF File), 56KB-Multimedia Appendix 1]

\section{Multimedia Appendix 2}

Questionnaire: perceived risk associated with the use of smart surveys. 
[PDF File (Adobe PDF File), 64KB-Multimedia Appendix 2]

\section{Multimedia Appendix 3}

In-depth interview (Think Aloud) prompts.

[PDF File (Adobe PDF File), 69KB-Multimedia Appendix 3]

\section{References}

1. Richards T, Montori VM, Godlee F, Lapsley P, Paul D. Let the patient revolution begin. Br Med J 2013 May 14;346:f2614. [doi: 10.1136/bmj.f2614] [Medline: 23674136]

2. Boulding W, Glickman SW, Manary MP, Schulman KA, Staelin R. Relationship between patient satisfaction with inpatient care and hospital readmission within 30 days. Am J Manag Care 2011 Jan;17(1):41-48 [FREE Full text] [Medline: 21348567]

3. Doyle C, Lennox L, Bell D. A systematic review of evidence on the links between patient experience and clinical safety and effectiveness. BMJ Open 2013 Jan 3;3(1) [FREE Full text] [doi: 10.1136/bmjopen-2012-001570] [Medline: 23293244]

4. Ahmed F, Burt J, Roland M. Measuring patient experience: concepts and methods. Patient 2014;7(3):235-241. [doi: 10.1007/s40271-014-0060-5] [Medline: 24831941]

5. Price RA, Elliott MN, Zaslavsky AM, Hays RD, Lehrman WG, Rybowski L, et al. Examining the role of patient experience surveys in measuring health care quality. Med Care Res Rev 2014 Oct;71(5):522-554 [FREE Full text] [doi: 10.1177/1077558714541480] [Medline: 25027409]

6. McMurray J, McNeil H, Lafortune C, Black S, Prorok J, Stolee P. Measuring patients' experience of rehabilitation services across the care continuum. Part I: a systematic review of the literature. Arch Phys Med Rehabil 2016 Jan;97(1):104-120. [doi: 10.1016/j.apmr.2015.08.407] [Medline: 26299752]

7. Miller G. The smartphone psychology manifesto. Perspect Psychol Sci 2012 May;7(3):221-237. [doi: 10.1177/1745691612441215] [Medline: 26168460]

8. Tomlinson M, Solomon W, Singh Y, Doherty T, Chopra M, Ijumba P, et al. The use of mobile phones as a data collection tool: a report from a household survey in South Africa. BMC Med Inform Decis Mak 2009;9:51 [FREE Full text] [doi: 10.1186/1472-6947-9-51] [Medline: 20030813]

9. McMurray J, McNeil H, Gordon A, Elliott J, Stolee P. Building a rehabilitative care measurement instrument to improve the patient experience. Arch Phys Med Rehabil 2019 Jan;100(1):39-44. [doi: 10.1016/j.apmr.2018.05.022] [Medline: 29958901]

10. McMurray J, McNeil H, Gordon A, Elliott J, Stolee P. Psychometric testing of a rehabilitative care patient experience instrument. Arch Phys Med Rehabil 2018 Sep;99(9):1840-1847. [doi: 10.1016/j.apmr.2018.04.028] [Medline: 29852150]

11. WatLX. URL: https://uwaterloo.ca/watlx/ [accessed 2018-01-23] [WebCite Cache ID 6wh1DseTq]

12. Abernethy AP, Herndon JE, Wheeler JL, Patwardhan M, Shaw H, Lyerly HK, et al. Improving health care efficiency and quality using tablet personal computers to collect research-quality, patient-reported data. Health Serv Res 2008 Dec;43(6):1975-1991 [FREE Full text] [doi: 10.1111/j.1475-6773.2008.00887.x] [Medline: 18761678]

13. Green AS, Rafaeli E, Bolger N, Shrout PE, Reis HT. Paper or plastic? Data equivalence in paper and electronic diaries. Psychol Methods 2006 Mar;11(1):87-105. [doi: 10.1037/1082-989X.11.1.87] [Medline: 16594769]

14. Marcano BJ, Jamsek J, Huckvale K, O'Donoghue J, Morrison CP, Car J. Comparison of self-administered survey questionnaire responses collected using mobile apps versus other methods. Cochrane Database Syst Rev 2015 Jul 27(7):MR000042. [doi: 10.1002/14651858.MR000042.pub2] [Medline: 26212714]

15. Ludford PJ, Frankowski D, Reily K, Wilms K, Terveen L. Because I Carry My Cell Phone Anyway: Functional Location-based Reminder Applications. In: Proceedings of the SIGCHI Conference on Human Factors in Computing Systems. USA: ACM; 2006 Presented at: CHI'06; April 22-27, 2006; Montreal, Canada p. 889-898 URL: https://dl.acm.org/ citation.cfm?id=1124903

16. Wang Y, Perez-Quinones M. Beyond "Geofencing": Specifying Location in Location-Based Reminder Applications. In: Proceedings of the 33rd Annual ACM Conference Extended Abstracts on Human Factors in Computing Systems. USA: ACM; 2015 Presented at: CHI EA'15; April 18-23, 2015; Seoul, South Korea p. 1767-1772.

17. Pielot M, Church K, de Oliveira R. An In-situ Study of Mobile Phone Notifications. In: Proceedings of the 16th International Conference on Human-Computer Interaction with Mobile Devices \& Services. 2014 Presented at: MobileHCI'14; September 23-26, 2014; Toronto, Canada p. 233-242.

18. Sullivan M, Bornstein S, McMurray J. Memorial University of Newfoundland. 2016. The Effectiveness of Digital Surveys for Collecting Patient Feedback URL: https://www.nlcahr.mun.ca/CHRSP/PATIENT FEEDBACK RER.pdf [accessed 2019-01-31] [WebCite Cache ID 75qCUNMH6]

19. Kuntsche E, Labhart F. Using personal cell phones for ecological momentary assessment. Eur Psychol Internet 2013;18(1):3-11. [doi: 10.1027/1016-9040/a000127]

20. Smith H, Dinev T. Information privacy research: an interdisciplinary review. MIS Q 2011;35(4):989-1015 https://misq.org/information-privacy-research-an-interdisciplinary-review.html. 
21. Bauer RA. Consumer Behavior as Risk Taking. In: Dynamic Marketing for a Changing World, Proceedings of the 43rd Conference of the American Marketing Association. 1960 Presented at: 43rd Conference of the American Marketing Association; June 15-17, 1960; Chicago p. 389-398.

22. Jacoby J, Kaplan LB. Association for Consumer Research. 1972. The components of perceived risk URL: http://acrwebsite. org/volumes/12016/volumes/sv02/SV02 [accessed 2019-02-04] [WebCite Cache ID 75vXKmLE6]

23. Featherman MS, Pavlou PA. Predicting e-services adoption: a perceived risk facets perspective. Int J Hum Comput Stud 2003 Oct;59(4):451-474. [doi: 10.1016/S1071-5819(03)00111-3]

24. Littler D, Melanthiou D. Consumer perceptions of risk and uncertainty and the implications for behaviour towards innovative retail services: the case of internet banking. J Retail Cons Serv 2006 Nov;13(6):431-443. [doi: 10.1016/j.jretconser.2006.02.006]

25. Iglesias-Pradas S, Pascual-Miguel F, Hernández-García Á, Chaparro-Peláez J. Barriers and drivers for non-shoppers in B2C e-commerce: a latent class exploratory analysis. Comput Human Behav 2013;29(2):314-322. [doi: 10.1016/j.chb.2012.01.024]

26. Biucky ST, Abdolvand N, Harandi SR. The effects of perceived risk on social commerce adoption based on the tam model. Int J Electron Commer Stud 2017;8(2):196. [Medline: 12876581]

27. da Chen L. A model of consumer acceptance of mobile payment. Int J Mob Commun 2008;6(1):32. [doi: 10.1504/IJMC.2008.015997]

28. Lee MC. Factors influencing the adoption of internet banking: an integration of TAM and TPB with perceived risk and perceived benefit. Electron Commer Res Appl 2009 May;8(3):130-141. [doi: 10.1016/j.elerap.2008.11.006]

29. Tan M, Leo TS. Factors influencing the adoption of internet banking. J Assoc Inf Syst 2000;1:1-44 [FREE Full text]

30. Schnall R, Higgins T, Brown W, Carballo-Dieguez A, Bakken S. Trust, perceived risk, perceived ease of use and perceived usefulness as factors related to mHealth technology use. Stud Health Technol Inform 2015;216:467-471 [FREE Full text] [doi: 10.3233/978-1-61499-564-7-467] [Medline: 26262094]

31. Zhou T. Examining location-based services usage from the perspectives of Unified Theory of Acceptance and Use of Technology and privacy risk. J Electron Commer Res 2012;13(2):135-144 [FREE Full text]

32. Westin AF. Social and political dimensions of privacy. J Soc Issues 2003;59(2):431-453. [doi: 10.1111/1540-4560.00072]

33. Kumaraguru P, Cranor LF. Carnegie Mellon University. 2005. Privacy Indexes: A Survey of Westin's Studies URL: https:/ /www.cs.cmu.edu/ ponguru/CMU-ISRI-05-138.pdf [accessed 2019-02-04] [WebCite Cache ID 75vZLYgbZ]

34. Consolvo S, Smith IE, Matthews T, LaMarca A, Tabert J, Powledge P. Location disclosure to social relations: why, when, \& what people want to share. In: Proceedings of the SIGCHI Conference on Human Factors in Computing Systems. 2005 Presented at: CHI'05; March 29-April 01, 2015; Atlanta, USA p. 81-90. [doi: 10.1145/1054972.1054985]

35. King J. CyLab Usable Privacy and Security Laboratory. 2009. Taken Out of Context: An Empirical Analysis of Westin's Privacy Scale URL: https://cups.cs.cmu.edu/soups/2014/workshops/privacy/s1p1.pdf [accessed 2019-01-31] [WebCite Cache ID 75qEImNSt]

36. Woodruff A, Pihur V, Consolvo S, Schmidt L, Brandimarte L, Acquisti A. Would a privacy fundamentalist sell their DNA for $\$ 1000 \ldots$ if nothing bad happened as a result? The Westin categories, behavioral intentions, and consequences. In: Proceedings of the Tenth Symposium on Usable Privacy and Security. 2014 Presented at: SOUPS 2014; July 9-11, 2014; Menlo Park, USA.

37. Dupree JL, Devries R, Berry DM, Lank E. Privacy personas: Clustering users via attitudes and behaviors toward security practices. In: Proceedings of the 2016 CHI Conference on Human Factors in Computing Systems. 2016 Presented at: CHI'16; May 7-12 2016; San Jose, USA p. 5228-5239. [doi: 10.1145/2858036.2858214]

38. Dupree JL, Lank E, Berry DM. A case study of using grounded analysis as a requirement engineering method: identifying personas that specify privacy and security tool users. Sci Comput Program 2018 Jan;152(C):1-37. [doi: 10.1016/j.scico.2017.08.010]

39. Morton A, Sasse M. Desperately seeking assurances: Segmenting users by their information-seeking preferences. 2014 Presented at: 2014 Twelfth Annual International Conference on Privacy, Security and Trust; July 23-24, 2014; Toronto, Canada p. 102-111. [doi: 10.1109/PST.2014.6890929]

40. Reinau KH, Harder H, Weber M. The SMS-GPS-Trip method: a new method for collecting trip information in travel behavior research. Telecomm Pol 2014;39(3-4):363-373. [doi: 10.1016/j.telpol.2014.05.006]

41. Kojo I, Heiskala M, Virtanen J. Customer journey mapping of an experience-centric service by mobile self-reporting: Testing the Qualiwall tool. 2014 Presented at: 16th International Conference on Human-Computer Interaction (HCII 2014); June 22-26, 2014; Crete, Greece.

42. Guest G, Bunce B, Johnson L. How many interviews are enough? An experiment with data saturation and variability. Field Methods 2006 Feb 1;18(1):59-82. [doi: 10.1177/1525822X05279903]

43. Morse JM. Determining sample size. Qual Health Res 2000;10(1):3-5. [doi: 10.1177/104973200129118183]

44. Metricwire Inc. URL: https://www.metricwire.com/ [accessed 2018-01-23] [WebCite Cache ID 6wh0n1Nxe]

45. Beatty PC, Willis GB. Research synthesis: the practice of cognitive interviewing. Public Opin Q 2007 Jun 5;71(2):287-311. [doi: $10.1093 / \mathrm{poq} / \mathrm{nfm} 006$ ] 
46. Sandelowski M. Reembodying qualitative inquiry. Qual Health Res 2002 Jan;12(1):104-115. [doi:

10.1177/1049732302012001008] [Medline: 11797919]

47. Fereday J, Muir-Cochrane E. Demonstrating rigor using thematic analysis: a hybrid approach of inductive and deductive coding and theme development. Int J Qual Methods 2016 Nov 29;5(1):80-92. [doi: 10.1177/160940690600500107]

48. Corbin J, Strauss A. Basics Of Qualitative Research: Techniques And Procedures For Developing Grounded Theory. Thousand Oaks, USA: Sage Publications; 2015.

49. Creswell JW. Research Design: Qualitative, Quantitative and Mixed Methods Approaches. Thousand Oaks, California: Sage Publications Inc; 2003.

50. Gkioulos V, Wangen G, Katsikas S, Kavallieratos G, Kotzanikolaou P. Security awareness of the digital natives. Information 2017 Apr 8;8(2):42. [doi: 10.3390/info8020042]

51. Martínez-Pérez B, de la Torre-Díez I, López-Coronado M. Privacy and security in mobile health apps: a review and recommendations. J Med Syst 2015 Jan;39(1):181. [doi: 10.1007/s10916-014-0181-3] [Medline: 25486895]

52. Braun R, Catalani C, Wimbush J, Israelski D. Community health workers and mobile technology: a systematic review of the literature. PLoS One 2013;8(6):e65772 [FREE Full text] [doi: 10.1371/journal.pone.0065772] [Medline: 23776544]

53. Free C, Phillips G, Galli L, Watson L, Felix L, Edwards P, et al. The effectiveness of mobile-health technology-based health behaviour change or disease management interventions for health care consumers: a systematic review. PLoS Med 2013;10(1):e1001362 [FREE Full text] [doi: 10.1371/journal.pmed.1001362] [Medline: 23349621]

54. Ashar R, Lewis S, Blazes D, Chretien J. Applying information and communications technologies to collect health data from remote settings: a systematic assessment of current technologies. J Biomed Inform 2010 Apr;43(2):332-341 [FREE Full text] [doi: 10.1016/j.jbi.2009.11.009] [Medline: 19961957]

55. Boulos MG, Wheeler S, Tavares C, Jones R. How smartphones are changing the face of mobile and participatory healthcare: an overview, with example from eCAALYX. Biomed Eng Online 2011 Apr 5;10:24 [FREE Full text] [doi: 10.1186/1475-925X-10-24] [Medline: 21466669]

56. Bryce J, Fraser J. The role of disclosure of personal information in the evaluation of risk and trust in young peoples' online interactions. Comput Human Behav 2014 Jan;30:299-306. [doi: 10.1016/j.chb.2013.09.012]

57. Mirkovic J. DUO Research Archive. 2012 Jan. Usability, security and mobility for mobile devices in healthcare information systems URL: https://www.duo.uio.no/handle/10852/35700 [accessed 2019-01-31] [WebCite Cache ID 75qGL5XjE]

58. Becker M. Understanding users' health information privacy concerns for health wearables. In: Proceedings of the 51st Hawaii International Conference on System Sciences. 2018 Presented at: HCISS 2018; January 3-6, 2018; Hawaii, USA p. 3261 URL: https://scholarspace.manoa.hawaii.edu/bitstream/10125/50301/1/paper0414.pdf [doi: 10.24251/hicss.2018.413]

59. Gefen D, Karahanna E, Straub D. Trust and TAM in online shopping: an integrated model. MIS Q 2003;27(1):90. [doi: 10.2307/30036519]

60. Kim DJ, Ferrin DL, Rao HR. A trust-based consumer decision-making model in electronic commerce: the role of trust, perceived risk, and their antecedents. Decis Support Syst 2008 Jan;44(2):544-564. [doi: 10.1016/j.dss.2007.07.001]

61. Tene O, Polonetsky J. Stanford Law Review. 2010. Privacy in the age of big data: a time for big decisions URL: https:/ /www.stanfordlawreview.org/online/privacy-paradox-privacy-and-big-data/ [accessed 2019-01-31] [WebCite Cache ID 75qLo51C9]

62. Barkuhus L, Dey AD. Location-Based Services for Mobile Telephony: A Study of Users' Privacy Concerns. 2003 Presented at: IFIP TC13 International Conference on Human-Computer Interaction; September 1-5, 2003; Zurich, Switzerland URL: http://citeseerx.ist.psu.edu/viewdoc/download?doi=10.1.1.10.527\&rep=rep1\&type=pdf

63. Venkatesh V, Davis FD. A theoretical extension of the technology acceptance model: four longitudinal field studies. Manage Sci 2000 Feb;46(2):186-204. [doi: 10.1287/mnsc.46.2.186.11926]

64. Venkatesh V, Morris M. User acceptance of information technology: toward a unified view. MIS Q 2003;27(1):18 [FREE Full text] [doi: 10.2307/30036540]

65. Canadian Institute for Health Information. 2014. National Health Expenditure Trends, 1975 to 2014 URL: http://www. cihi.ca/web/resource/en/nhex_2014_report_en.pdf [accessed 2019-01-31] [WebCite Cache ID 75qCdxf6C]

66. Pew Research Center. 2018. Mobile Fact Sheet Internet URL: http://www.pewinternet.org/fact-sheet/mobile/ [accessed 2018-11-02] [WebCite Cache ID 73dV8SG8t]

\section{Abbreviations}

GPS: Global Positioning System

IS: information system

mHealth: mobile health

PAS: privacy and security 
Edited by G Eysenbach; submitted 24.01.18; peer-reviewed by J Burkell, M Bardus, C Chen, A Paglialonga; comments to author 14.08.18; revised version received 13.11.18; accepted 06.01.19; published 18.03.19

Please cite as:

$\mathrm{Ng}$ D, McMurray J, Wallace J, Morita P

What Is Being Used and Who Is Using It: Barriers to the Adoption of Smartphone Patient Experience Surveys JMIR Form Res 2019;3(1):e9922

URL: http://formative.jmir.org/2019/1/e9922/

doi: 10.2196/formative.9922

PMID: 30882354

CDenise Ng, Josephine McMurray, James Wallace, Plinio Morita. Originally published in JMIR Formative Research (http://formative.jmir.org), 18.03.2019. This is an open-access article distributed under the terms of the Creative Commons Attribution License (https://creativecommons.org/licenses/by/4.0/), which permits unrestricted use, distribution, and reproduction in any medium, provided the original work, first published in JMIR Formative Research, is properly cited. The complete bibliographic information, a link to the original publication on http://formative.jmir.org, as well as this copyright and license information must be included. 